\title{
ON TOPOLOGICALLY AND QUASICONFORMALLY HOMOGENEOUS CONTINUA
}

\author{
BEVERLY BRECHNER ${ }^{1}$ ) and TIMO ERKAMA
}

A subset $M$ of the Riemann sphere is called quasiconformally homogeneous if for each pair of points $P$ and $Q$ of $M$ there is a quasiconformal map $\varphi$ defined in a neighborhood of $M$ such that $\varphi(M)=M$ and $\varphi(P)=Q$. For information about quasiconformal mappings, see [1].

Recently the second author showed [3] that a simple closed curve is quasiconformally homogeneous if and only if it is a quasicircle (i.e., the image of a circle under a quasiconformal map). In this note we prove the following more general result.

Theorem 1. Every non-degenerate quasiconformally homogeneous continuum is a quasicircle.

Note that a continuum is called non-degenerate if it is an infinite proper subset of the sphere.

It can be shown by function theoretic methods that a non-degenerate quasiconformally homogeneous continuum must contain an arc. Hence by a theorem of Bing [2] such a continuum is a simple closed curve. However, we prefer an alternative method which combines the result of [3] with a purely topological theorem.

Let $M$ be a proper subcontinuum of $S^{2}$. We say that $M$ is homogeneous with respect to neighborhood extensions if for each pair of points $x, y \in M$, there exist both a neighborhood $U$ of $M$ in $S^{2}$ and a homeomorphism $h: U \rightarrow S^{2}$ such that (1) $h(x)=y$ and (2) $h(M)=M$.

By definition, every quasiconformally homogeneous continuum is homogeneous with respect to neighborhood extensions. Thus Theorem 1 follows by Theorem 2 and [3].

Theorem 2. Let $M$ be a non-degenerate proper subcontinuum of $S^{2}$ such that $M$ is homogeneous with respect to neighborhood extensions. Then $M$ is a simple closed curve.

1) The first author is indebted to Morton Brown and F. W. Gehring, both for bringing this problem to her attention and for a number of interesting conversations. 
Proof.

(1) Clearly each point of $M$ must be accessible.

(2) Any indecomposable plane continuum contains inaccessible points by [5].

(3) Thus $M$ contains no indecomposable continuum and is hereditarily decomposable.

(4) Now by Theorem 2 of [4], every homogeneous hereditarily decomposable plane continuum is a simple closed curve.

The theorem follows.

\section{References}

[1] Bers, Lipman: Quasiconformal mappings with applications to differential equations, function theory, and topology. - Bull. Amer. Math. Soc. 83, 1977, 1083-1100.

[2] Bing, R. H.: A simple closed curve is the only homogeneous bounded plane continuum that contains an arc. - Canad. J. Math. 12, 1960, 209-230.

[3] Erkama, T.: Quasiconformally homogeneous curves. - Michigan Math. J. 24, 1977, 157-159.

[4] Hagopian, C. L.: Homogeneous plane continua. - Houston J. Math. 1, 1975, 35-41.

[5] Mazurkiewicz, S.: Sur les points accessibles des continus indécomposables. - Fund. Math. 14, 1929, 107-115.

University of Florida

Department of Mathematics

Gainesville, Florida 32611

USA

Received 23 October 1978
University of Helsinki

Department of Mathematics SF-00100 Helsinki 10

Finland 